\title{
AIRES: An Airborne Infra-Red Echelle Spectrometer for SOFIA
}

Jessie L. Dotson ${ }^{1}$, Edwin F. Erickson, Michael R. Haas, Sean W. J. Colgan, and Janet P. Simpson ${ }^{1}$

NASA Ames Research Center, MS 245-6, Moffett Field, CA 94035

Charles M. Telesco and Robert K. Piña

University of Florida

Jürgen Wolf

DLR-WS, Berlin

Erick T. Young

University of Arizona

Abstract. SOFIA will enable astronomical observations with unprecedented angular resolution at infrared wavelengths obscured from the ground. To help open this new chapter in the exploration of the infrared universe, we are building AIRES, an Airborne Infra-Red Echelle Spectrometer. AIRES will be operated as a first generation, general purpose facility instrument by USRA, NASA's prime contractor for SOFIA. AIRES is a long slit spectrograph operating from $17-210 \mu \mathrm{m}$. In high resolution mode the spectral resolving power is $\sim 10^{6} \mu \mathrm{m} / \lambda$ or $\sim 10^{4}$ at $100 \mu \mathrm{m}$. Unfortunately, since the conference, a low resolution mode with resolving power about 100 times lower has been deleted due to budgetary constraints. AIRES includes a slit viewing camera which operates in broad bands at 18 and $25 \mu \mathrm{m}$.

\section{Introduction}

AIRES will be ideal for spectral imaging of gas-phase phenomena in the interstellar medium (ISM). Far-infrared line observations probe the excitation, pressure, density, luminosity, chemical composition, heating and cooling rates, mass distribution, and kinematics in the various components of the ISM. The lines offer invaluable and often unique diagnostics of conditions in such diverse objects as star forming regions, circumstellar shells, the Galactic Center, starbursts in galaxies, and the nuclei of active galaxies. AIRES will provide new insights into these and other environments in the ISM. It will also be useful for

${ }^{1}$ SETI Institute

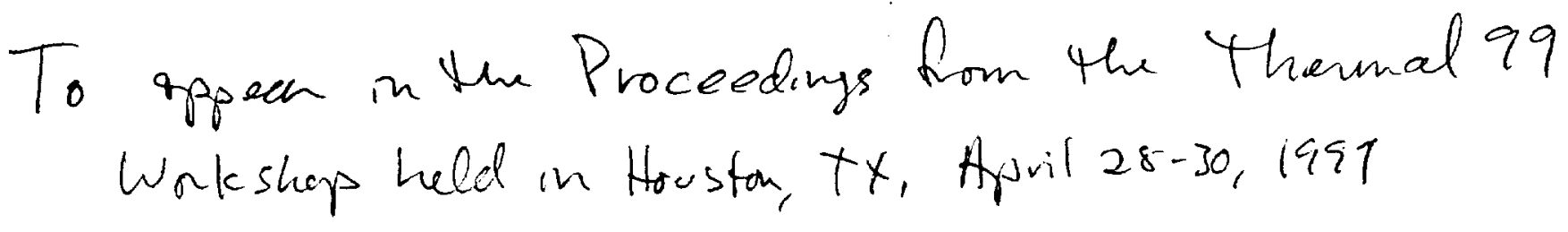


studies of solar system phenomena such as planetary atmospheres, comets, and the sun.

AIRES' low resolution mode would have enabled comparison of infrared continuum emission with line emission from interstellar clouds whose continuum is too faint to be measured in high resolution mode. Such observations, as well as measurements of long wavelength features of interstellar grains and solid surfaces of solar system bodies will need to be made with other instruments.

\section{Observing with SOFIA}

SOFIA, a $2.7 \mathrm{~m}$ telescope installed in a converted Boeing $747 \mathrm{SP}$, is a joint project between NASA and DLR (the German aerospace research establishment). USRA is the prime contractor for development and will operate the observatory for NASA. Science observations are scheduled to begin in late 2002.

From its operating altitude of $\sim 41,000$ feet, SOFIA will avoid more than $99 \%$ of the obscuring water vapor, allowing observations at wavelengths (particularly in the far-infrared) which are inaccessible from the ground (see Figure 1). At higher spectral resolutions, the atmospheric transmission is interrupted by a forest of narrow absorption lines due to telluric $\mathrm{O}_{3}, \mathrm{CO}_{2}$, and $\mathrm{H}_{2} \mathrm{O}$ (see inset of Figure 1). The effects of these atmospheric absorption lines can effectively be removed from spectroscopic observations, with a corresonding reduction in $\mathrm{S} / \mathrm{N}$, as long as they are unsaturated (e.g. Burton \& Haas 1997).

\section{Instrument}

AIRES is a long slit spectrograph operating from $17-210 \mu \mathrm{m}$. The instrument's expected performance is summarized in Table 1 . A cross section of the instrument mounted on the telescope is shown in Figure 2. The cryostat, mechanical and optical systems are in the detailed design phase. A ray trace of the optical system is shown in Figure 3. A K-mirror allows any orientation of the slit on the sky. The slit width is selectable. Fabrication of the 42 inch long echelle grating has commenced because it is a long lead time, technically challenging, critical component of the system. AIRES has 4 detector arrays; one for the slit viewer and 3 to span the entire spectroscopic wavelength range as specified in Table 2. Prototypes for the two long wavelength spectroscopy arrays are being tested at NASA Ames Research Center. The short wavelength spectroscopy array is in fabrication at Boeing. The array for the slit viewer has been purchased from Boeing. We will begin testing the slit viewer array at University of Florida in August 1999.

\section{Astrophysics with AIRES}

Some far infrared spectral features are listed in Table 3. The atomic and molecular features are candidates for observation with AIRES. Features so far observed in both emission and absorption are indicated with a/e. Whereas the dust features typically originate in regions cooler than a few hundred degrees Kelvin, 


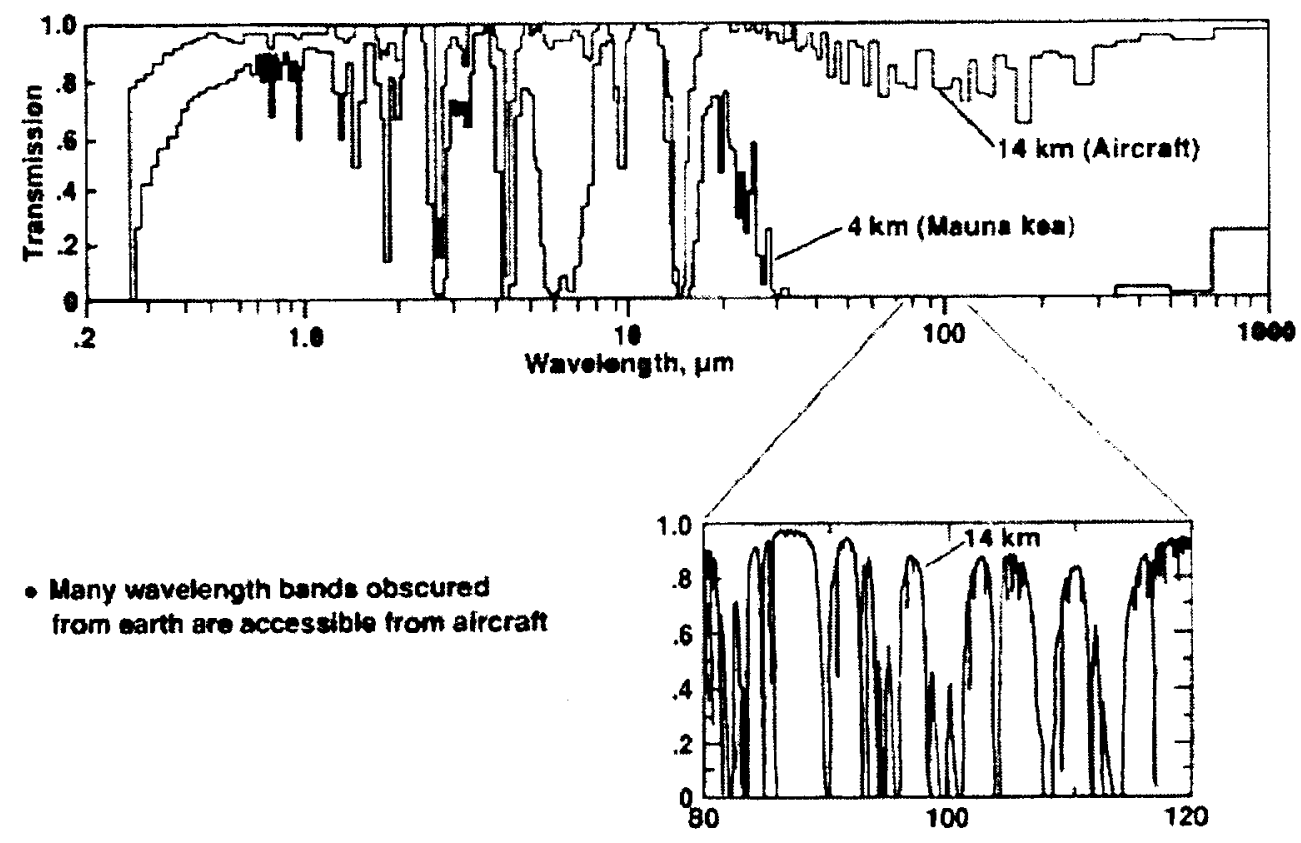

Figure 1. Atmospheric transmission at Mauna Kea and at SOFIA's altitude (from Erickson 1998). The model assumes a vertical precipitable column depth of water $\mathrm{w}=2.3 \mu \mathrm{m}$ at $14 \mathrm{~km}$ altitude, $\mathrm{w}=1.2$ $\mathrm{mm}$ at $4 \mathrm{~km}$ altitude, and an elevation angle of $40^{\circ}$.

Table 1. AIRES Attributes

\begin{tabular}{|c|c|}
\hline Spatial Resolution & $1.2 \lambda / \mathrm{D} \sim(\lambda / 10 \mu \mathrm{m})^{\prime \prime}$ pixels; e.g. $10^{\prime \prime}$ at $100 \mu \mathrm{m}$ \\
\hline Slit Orientation & Selectable with cryogenic K-mirror \\
\hline Wavelength / Mode Changes & $<1$ minute \\
\hline Spectroscopy & \\
\hline $\begin{array}{l}\text { Wavelength Coverage } \\
\text { Slit Size }\end{array}$ & $\begin{array}{l}\lambda=17-210 \mu \mathrm{m} \\
2.5^{\prime} \text { long with width variable from } 2^{\prime \prime} \text { to } 35^{\prime \prime}\end{array}$ \\
\hline $\begin{array}{l}\text { Detector Arrays } \\
\quad \text { (spatial x spectral) }\end{array}$ & $\begin{array}{l}17-40 \mu \mathrm{m}: 128 \times 128, \sim 1.3^{\prime \prime} \text { pixels (Si:Sb BIB) } \\
40-120 \mu \mathrm{m}: 24 \times 16, \sim 7^{\prime \prime} \text { pixels (Ge:Sb PC) } \\
120-210 \mu \mathrm{m}: 12 \times 8, \sim 14^{\prime \prime} \text { pixels (Ge:Ga stressed PC) }\end{array}$ \\
\hline $\begin{array}{l}\text { Spectral Resolution } \\
\text { Free Spectral Range } \\
\text { Line Sensitivity }\end{array}$ & $\begin{array}{l}\mathrm{R}=\lambda / \Delta \lambda \sim 10^{6} \mu \mathrm{m} / \lambda \\
\Delta \mathrm{v} \sim 200-350 \mathrm{~km} / \mathrm{s} \\
\sim 1-5 \times 10^{-21} \mathrm{~W} / \mathrm{cm}^{2}(4 \sigma, 1 \mathrm{hr})\end{array}$ \\
\hline $\begin{array}{l}\text { Imaging / Slit Viewing } \\
\text { Wavelength Bands } \\
\text { Spectral Resolution } \\
\text { Field of View } \\
\text { Detector Array } \\
\text { Continuum Sensitivity }\end{array}$ & $\begin{array}{l}18,25 \mu \mathrm{m} \\
\mathrm{R}=\lambda / \Delta \lambda \sim 5 \\
2.5^{\prime} \times 2.5^{\prime}\left(\text { with } 2^{\prime \prime} \text { slit); } 1.0^{\prime} \times 2.5^{\prime} \text { (wider slits) }\right. \\
128 \times 128, \sim 1.3^{\prime \prime} \text { pixels (Si:As BIB) } \\
\sim 15 \mathrm{mJy}(4 \sigma, 1 \mathrm{hr})\end{array}$ \\
\hline
\end{tabular}




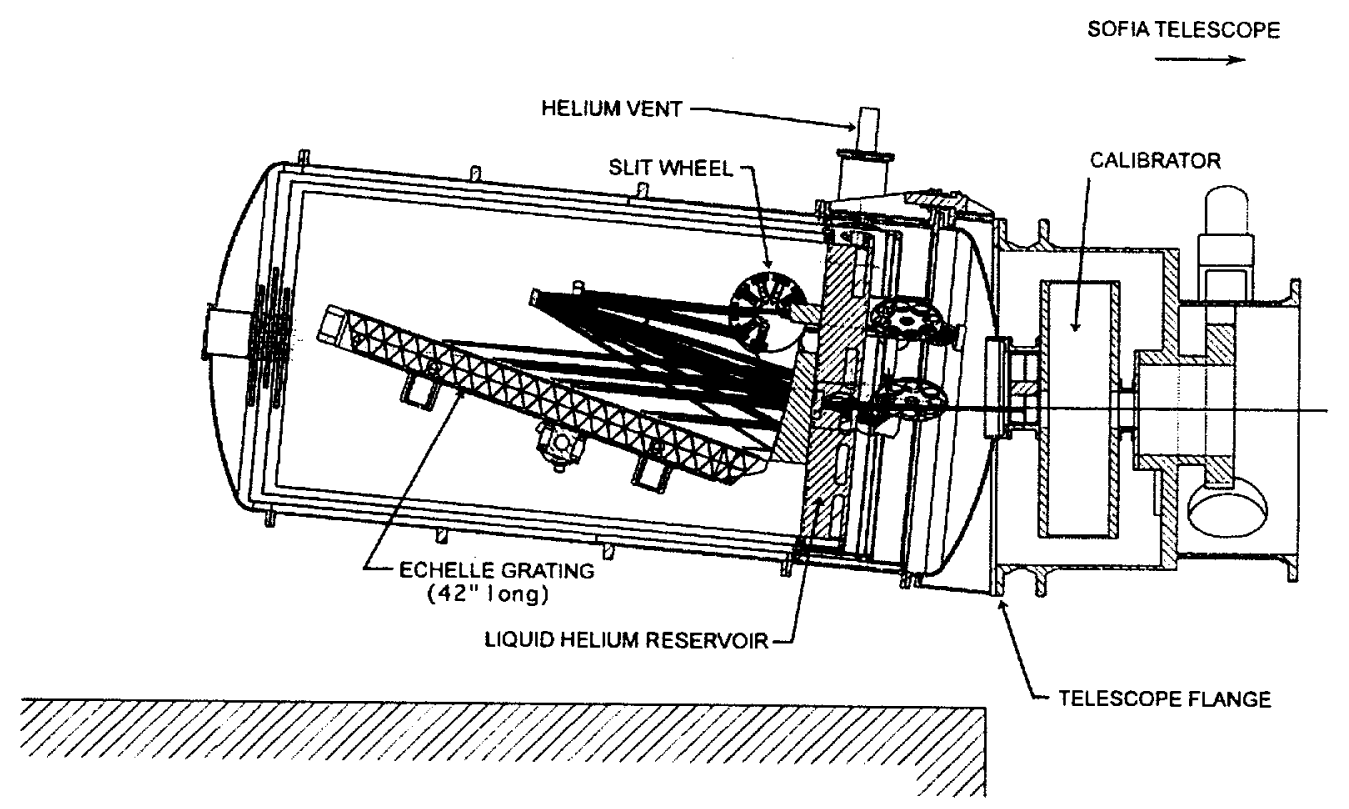

Figure 2. A cross section of AIRES is shown mounted on the SOFIA telescope. The large echelle grating disperses light for high resolution spectroscopy. The entire optical system is cooled to $4 \mathrm{~K}$ with liquid helium.

Table 2. AIRES Detector Characterisitics

\begin{tabular}{cccccc}
\hline $\begin{array}{c}\text { Wavelength } \\
\text { Range } \\
(\mu \mathrm{m})\end{array}$ & Material & Type & Mode & Format & Pitch \\
\hline $17-28$ & & & & & \\
$17-42$ & Si:As & BIB & Imaging & $128 \times 128$ & 0.75 \\
$42-125$ & Ge:Sb & BIB & Spectroscopy & $128 \times 128$ & 0.75 \\
$125-210$ & Ge:Ga & PC & Spectroscopy & $24 \times 16$ & 2 \\
\hline \hline
\end{tabular}




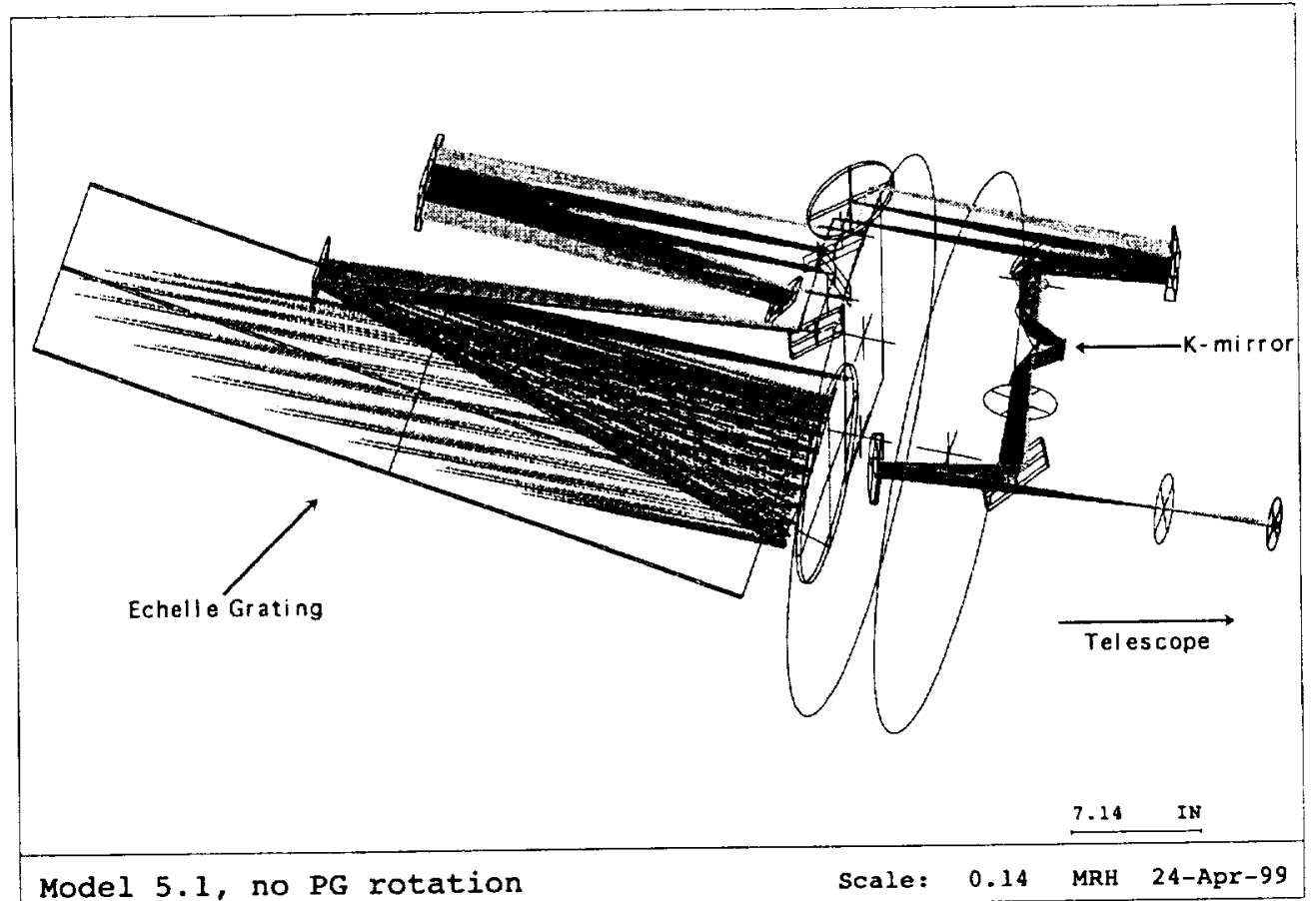

Figure 3. A ray trace of the AIRES optical system. All reflecting optics are made of aluminum and are cooled to $4 \mathrm{~K}$. The light enters from the telescope on the lower right hand side of the figure. The foreoptics (right hand side) contain the K-mirror assembly and the blocking filters. Next the light passes through the slit wheel to the predisperser assembly (upper left). The predisperser gratings operate in first order and sort orders for the echelle grating. After passing through a second slit, the light enters the echelle assembly which consists of the echelle grating and its collimator. The echelle grating provides the high resolution dispersion. 
the various gaseous lines originate in regions ranging in temperature from $10^{5} \mathrm{~K}$ down to $20 \mathrm{~K}$.

Table 3. Some Far-Infrared Spectral Features

\begin{tabular}{|c|c|c|c|c|c|}
\hline Atoms & & \multicolumn{2}{|l|}{ Molecules } & \multicolumn{2}{|c|}{ Solid State Features } \\
\hline Wavelength $(\mu \mathrm{m})$ & Species & Wavelength $(\mu \mathrm{m})$ & Species & Wavelength $(\mu \mathrm{m})$ & Species \\
\hline $52.5,88.8,169.4$ & HI & $77,84,97,103$ & CO & $19.0 \mathrm{a} / \mathrm{e}$ & Silicate \\
\hline 21.8 & Ar III & $119,124,153,163$ & & $23.6,33.6,69$ & Olivine \\
\hline $17.9,26.0$ & $\mathrm{Fe}$ II & 186,200 & & $26.2,32.9,35.8$ & Crystalline Pyroxene \\
\hline $18.7,33.4$ & S III & 17.0 & $\mathrm{H}_{2}$ & $40.6,43.1$ & \\
\hline 22.9 & Fe III & $53.4,84.4,84.69$ & $\mathrm{OH}$ & 44 & $\mathrm{H}_{2} \mathrm{O}$ Ice \\
\hline $\begin{array}{l}24.3 \\
25.2\end{array}$ & $\begin{array}{l}\text { Ne VI } \\
\text { S I }\end{array}$ & $\begin{array}{l}119.3,119.5,149 \\
163.1,164.4,202 \mathrm{a} / \mathrm{e}\end{array}$ & & & \\
\hline 25.9 & O IV & 154 & ${ }^{13} \mathrm{CO}$ & & \\
\hline 34.8 & Si II & $120.0,120.2 \mathrm{a} / \mathrm{e}$ & ${ }^{18} \mathrm{OH}$ & & \\
\hline 36.0 & $\mathrm{Ne}$ III & 125,166 & $\mathrm{NH}_{3}$ & & \\
\hline $51.8,88.4$ & O III & $149.1,149.4,203$ & $\mathrm{CH}$ & & \\
\hline 57.3 & N III & & & & \\
\hline $\begin{array}{l}63.2,146 \\
121.9,205\end{array}$ & O I & & & & \\
\hline 158 & C II & & & & \\
\hline
\end{tabular}

To begin to appreciate the value of spectroscopy in this wavelength band, consider the fine structure lines listed in Table 4. These low lying transitions are quite insensitive to gas temperature and span a wide range of ionization potential (I.P.) and critical density $\left(\mathrm{N}_{\text {crit }}\right)$. The line pairs with a common energy level (e.g., [O I] 63, $146 \mu \mathrm{m}$; [N II] 122, $205 \mu \mathrm{m}$; [O III] $52,88 \mu \mathrm{m}$; and [S III] 19, 33 $\mu \mathrm{m})$ serve as density probes, whereas line pairs from different ionization states (e.g., [N II] $122 \mu \mathrm{m}$ and [N III] $57 \mu \mathrm{m}$; [O I] $63 \mu \mathrm{m}$, [O III] $52 \mu \mathrm{m}$, and [O IV] $26 \mu \mathrm{m} ;$ [Ne III] $36 \mu \mathrm{m}$ and [Ne V] $24 \mu \mathrm{m}$ ) can be used to obtain excitation conditions (e.g. Colgan et al. 1993). With some assumptions about relative abundances, but weak dependence on density, the [S III] $33 \mu \mathrm{m}-[\mathrm{O}$ III] $52 \mu \mathrm{m}$ line pair provides a valuable alternative estimate of the excitation conditions in $\mathrm{H}$ II regions (e.g. Rubin et al. 1994). Lines with similar ionization potentials (e.g., [N III] $57 \mu \mathrm{m}$ and [O III] $52 \mu \mathrm{m}$; [Fe II] $26 \mu \mathrm{m}$, [Si II] $35 \mu \mathrm{m}$, and [O I] 63 $\mu \mathrm{m})$ can be used to examine relative abundances.

Some lines serve as signposts of a particular emission mechanism. For example, [S I] $25 \mu \mathrm{m}$ originates primarily from fast, high-density wind shocks (Hollenbach \& McKee 1989) and can be used to distinguish these from overlying Photo-Dissociation Regions (PDRs) (Tielens \& Hollenbach 1985a, 1985b). The strength of the low excitation lines compared to the associated FIR continuum luminosity also helps to distinguish shock emission (which produces little continuum luminosity) from PDR emission (normally associated with significant FIR continuum). The [C II] $158 \mu \mathrm{m},[\mathrm{O}$ I] $63,146 \mu \mathrm{m}$ line fluxes and the total FIR continuum flux allow estimates of the relative importance of gas and dust cooling, the atomic density, and the incident UV radiation field in PDRs (Wolfire et al. 1990). The combination of [S I] $25 \mu \mathrm{m},[\mathrm{Fe} \mathrm{II}] 26 \mu \mathrm{m}$, [Si II] $35 \mu \mathrm{m}$, and [O I] $63 \mu \mathrm{m}$ yield the shock density, velocity, and mass loss in fast wind shocks. Similarly, the line fluxes in FIR molecular transitions such as $\mathrm{H}_{2} 17 \mu \mathrm{m}$, high-J $\mathrm{CO}$ and $\mathrm{H}_{2} \mathrm{O}$ can be combined to estimate the density and velocity of slower $\left(\mathrm{v}_{s} \leq 50 \mathrm{~km} / \mathrm{s}\right)$ shocks. The FIR continuum distribution can reveal the luminosity of many abscured sources, and contains spectral features characterizing ices and minerals in interstellar grains. 
In sum, the FIR observations probe the pressures, densities, luminosities, excitation, mass distribution, chemistry, and dominant heating and cooling rates in the various components of the ISM. Thus the lines offer invaluable and often unique diagnostics of conditions in such diverse places as stellar nurseries, circumstellar shells, the Galactic center, starbursts in galaxies, and the nuclei of active galaxies. For illustration purposes, two examples are discussed below.

Table 4. FIR Fine-Structure Lines

\begin{tabular}{lrcc}
\hline Species & $\begin{array}{c}\lambda \\
(\mu \mathrm{m})\end{array}$ & $\begin{array}{c}\operatorname{LogN}_{\text {crit }}^{\dagger} \\
\left(\mathrm{cm}^{-3}\right)\end{array}$ & $\begin{array}{c}\text { I.P. Range } \\
(\mathrm{eV})\end{array}$ \\
\hline Fe II & 17.9 & 4.7 & $7.9-16.2$ \\
S III & 18.7 & 4.1 & $23.4-35.0$ \\
Fe III & 22.9 & 4.8 & $16.2-30.6$ \\
Fe I & 24.0 & 6.5 & $0.0-7.9$ \\
Ne V & 24.3 & 4.7 & $97.0-126.3$ \\
S I & 25.2 & 6.3 & $0.0-10.4$ \\
O IV & 25.9 & 4.0 & $54.9-77.4$ \\
Fe II & 26.0 & 6.3 & $7.9-16.2$ \\
S III & 33.5 & 3.3 & $23.4-35.0$ \\
Si III & 34.8 & 5.5 & $8.1-16.3$ \\
Ne III & 36.0 & 4.3 & $41.1-63.5$ \\
O III & 51.8 & 3.5 & $35.1-54.9$ \\
N III & 57.3 & 3.3 & $29.6-47.4$ \\
O I & 63.2 & 5.7 & $0.0-13.6$ \\
O III & 88.4 & 2.7 & $35.1-54.9$ \\
N II & 121.9 & 2.4 & $14.5-29.6$ \\
O I & 145.5 & 5.0 & $0.0-13.6$ \\
C II & 157.7 & 3.4 & $11.3-24.4$ \\
N II & 205.3 & 1.6 & $14.5-29.6$ \\
\hline
\end{tabular}

The critical density is for collisions with electrons (I.P. $>13.6 \mathrm{eV}$ ) or H-atoms (I.P. $<13.6$ $\mathrm{eV})$.

\subsection{High Resolution Spectroscopy}

AIRES is well suited to investigate the nature of outflows around high mass young stars. Although outflows from young stellar objects have been observed for two decades, there are still insufficient data to distinguish between competing theories for the origin of the winds which drive the outflows and to determine whether outflows limit the final stellar mass by halting the collapse of infalling material. Figure 4 shows a NICMOS image of Orion in $\mathrm{H}_{2}$ and $2.1 \mu \mathrm{m}$ continuum with overlaid [O I] $63 \mu \mathrm{m}$ contours. The $\mathrm{H}_{2}$ is believed to arise from a molecular shock; the [OI] from a wind shock. Wind shocks near the outflow source should be particularly strong in [S I] $25 \mu \mathrm{m}$, [Si II] $35 \mu \mathrm{m}$, and [Fe II] $26 \mu \mathrm{m}$. An image in these lines should allow identification of the outflow source to within $2-4^{\prime \prime}$. Observations at $63 \mu \mathrm{m}$ will explore the geometric relationship between the molecular and wind shocks. 

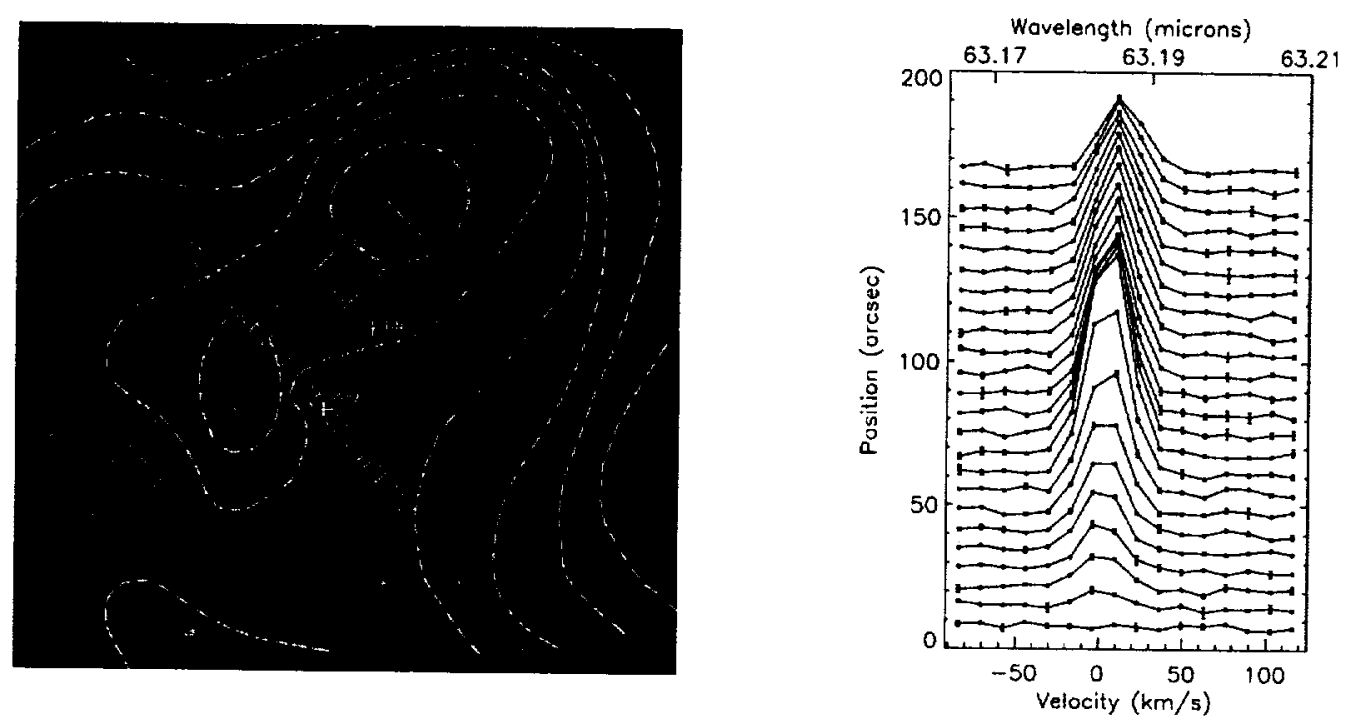

Figure $4 . \quad$ a) A NICMOS $\mathrm{H}_{2}$ and $2.1 \mu \mathrm{m}$ continuum image of Orion (Schultz et al. 1999) with superposed neutral oxygen [O I] $63 \mu \mathrm{m}$ contours (Werner et al. 1984). The $\mathrm{H}_{2}$ and reflected continuum show numerous clumps in the overall flow. The AIRES slit for $63 \mu \mathrm{m}\left(7^{\prime \prime}\right.$ across) is shown crossing the two [O I] peaks and the slit for $25 \mu \mathrm{m}$ (3" slit) is shown near the estimated location of the low velocity outflows. b) Synthetic spectra of the $63 \mu \mathrm{m}$ line as could be measured with AIRES. Depending on detector type, AIRES will measure 12, 24, or 128 spectra simultaneously from different locations along the slit. The $\mathrm{K}$-mirror (image rotator) will orient the slit at any position angle on the sky. 


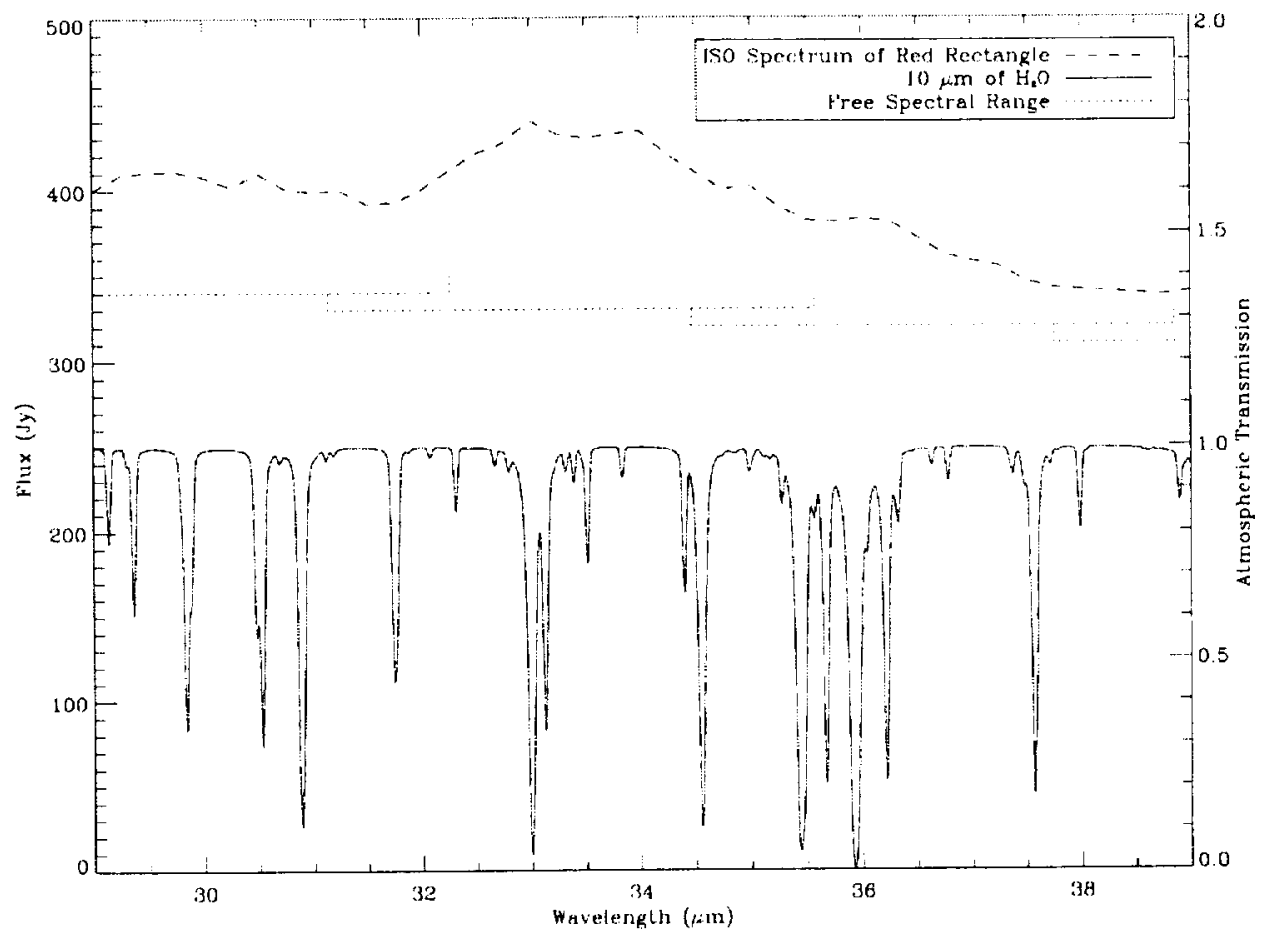

Figure 5. A portion of the spectrum of the Red Rectangle measured by ISO (Waters et al. 1998). AIRES' background limited noise in low resolution mode at $30 \mu \mathrm{m}$ will be $1 \mathrm{Jy}$ in 30 seconds $(1 \sigma)$. The free spectral range of AIRES in low resolution mode is shown by the dotted bars. There are 128 spectral resolution elements in each free spectral range. The silicate features between 33 and $35 \mu \mathrm{m}$ can be covered in one grating setting. The solid line is a model of the atmospheric transmission at SOFIA's altitude (assuming $10 \mu \mathrm{m}$ of precipitable water vapor). The effects of atmospheric transmission can be removed from the data when the absorption lines are not saturated. 


\subsection{Low Resolution Spectroscopy}

SOFIA's high spatial resolution makes it ideal for follow-up investigations of ISO observations. For example, ISO observations of circumstellar disks have produced a surge in the identification of solid state dust features (e.g. Tielens et al. 1998, Waters et al. 1998). A target which would benefit greatly from high spatial resolution observations is the Red Rectangle, a carbon rich reflection nebulae surrounding low-mass stars in the final stages of evolution. Waters et al. (1998) identified solid state spectral signatures of oxygen rich crystalline silicates in the Red Rectangle. On the basis of $11.3 \mu \mathrm{m}$ narrow band measurements (which are thought to be indicative of PAHs), they speculate that the carbonrich material is located in the extended nebula $\left(\sim 20^{\prime \prime} \times 10^{\prime \prime}\right)$ while the oxygenrich silicates are confined to the circumbinary disk. The ISO SWS spectrum of the Red Rectangle was obtained with a spatial resolution $\sim 20^{\prime \prime} \times 30^{\prime \prime}$. SOFIA's spatial resolution at $30 \mu \mathrm{m}$ will be $\sim 3^{\prime \prime}$. Low spectral resolution observations of the Red Rectangle, should confirm that the oxygen rich silicates are confined

to the cirumbinary disk.

Acknowledgments. AIRES is funded by a grant from the Universities Space Research Association for development of SOFIA science instruments. J. D. and J. S. acknowledge support from NASA/Ames Research Center Research Interchange Grant NCC2-647.

\section{References}

Burton, M. G., \& Haas, M. R. 1997, A\&A, 327, 309

Colgan, S. W. J., Haas, M. R., Erickson, E. F., Rubin, R. H., Simpson, J. P., \& Russel, R. W. 1993, ApJ, 413, 237

Erickson, E. F. 1998, PASP, 110, 1098

Haas, M. R., Hollenbach, D. J., \& Erickson, E. F. 1991, ApJ 374, 555

Hollenbach, D. J., \& McKee, C. F. 1989, ApJ, 342, 306

Rubin, R. H., Simpson, J. P., Lord, S. D., Colgan, S. W. J., Erickson, E. F., \& Haas, M. R. 1994, ApJ, 420, 772

Schultz, A. S. B., Colgan, S. W. J., Erickson. E. F., Kaufman, M. J., Hollenbach, D. J., O'Dell, C. R., Young, E. T., \& Chen, H. 1999, ApJ, 511, 282

Tielens, A. G. G. M., Waters, L. B. F. M., Molster, F. J., \& Justtanont, K. 1998, Ap\&SS, 255, 415

Tielens, A. G. G. M. \& Hollenbach, D. J. 1985a, ApJ, 291, 722

Tielens, A. G. G. M. \& Hollenbach, D. J. 1985a, ApJ, 291, 747

Waters, L.B.F.M., Cami,J., DeJong,T., Molster,F.J., VanLoon,J.Th., Bouwman,J., DeKoter,A., Waelkens,C., VanWinckel,H., \& Morris,P.W. 1998, Nature, 391,868

Werner, M. W., Hollenbach, D. J., Crawford, M. K., Genzel, R., Townes, C. H., \& Watson, D. M. 1984, ApJ, 282, L81

Wolfire, M. G., Tielens, A. G. G. M., \& Hollenbach, D. J. 1990, ApJ, 358, 116 